\title{
Non-Destructive Detection of Rebar Buried in a Reinforced Concrete Wall with Wireless Passive SAW Sensor
}

\author{
Yanping Fan $^{1}$, Xiaojun $\mathrm{Ji}^{1}$, Ping Cai ${ }^{1}$, Qianhui $\mathrm{Lu}^{2}$ \\ ${ }^{1}$ School of Electronics and Information, Shanghai Jiao Tong University, No.800 Dongchuan Road, \\ Minhang District, Shanghai, China \\ fypofcas@163.com; jxj127@sjtu.edu.cn (corresponding author); pcai@sjtu.edu.cn \\ ${ }^{2}$ Hangzhou Normal University, No.16 Xuelin Street, Xiasha Higher Education Zone, Hangzhou, Zhejiang, China
}

\begin{abstract}
In order to reduce the damage to the old reinforced concrete walls and work out the best construction scheme during the renovation of old buildings, it is often required to detect the position of rebar buried in concrete walls. In this paper, we propose a non-destructive method to detect the buried rebar by self-inductive sensor combined with surface acoustic wave resonator (SAWR). The proposed method has the advantages of wireless, passive and convenient operations. In our new design, the sensing element of self-inductance coil was made as a component of SAWR matching network. The distribution of rebar could be measured according to the system resonant frequency, using a signal demodulation device set. The depth of buried rebar and the deviation of output resonant frequency from inherent frequency of SAWR have an inverse relation. Finally, the validity of the method was verified in theoretical calculation and simulation.
\end{abstract}

Keywords: Reinforced concrete, rebar detection, passive wireless, non-destructive detection, surface acoustic wave resonator

\section{INTRODUCTION}

$I^{2}$ N ORDER to maintain the quality and reduce damage to the building structure, in rehabilitating historic buildings for old city renovation and protection, it is required to use a non-destructive method to clearly mark where the rebar was buried in concrete walls to avoid being cut, such as the method of avoiding mechanical contact [1]. There are many non-destructive methods to test the parameters of reinforced concrete walls, such as the ultrasonic method, sclerometric methods and the pull-out method [2]. The traditional method for non-destructive rebar detection is to measure the small change of excited magnetic field by many kinds of magnetic sensors [3]-[5]. Although this method has the advantage of relative maturity, it suffers from the complexity of signal processing, requirement of bulk measuring equipment, large intensity of operation, the difficulty of remote control and so on. Reference [6] presented a method to make use of eddy current effect for detection of the rebar embedded in concrete walls by measuring the inductance change of a solenoid coil caused by the eddy current and magnetization. This method had the same limitation as the electromagnetic method and consumed huge amounts of energy when the testing equipment was operating. Surface acoustic wave (SAW) device is a kind of passive device, which can also work at the wireless mode. An effective wireless passive SAW non-destructive sensor was developed to detect the rebar buried in concrete walls by combining the conventional self-inductive sensing technique with the SAW technique. The sensing self-inductance was integrated into the matching-network of SAWR system [7]. It separates the sensing element from the signal control and processing elements, and thus possesses the capability of remote sensing. It also has the advantages of small size, light weight, and easy handling. Moreover, it can work under the conditions of high temperature, high pressure, in dusty, toxic and other harmful environment. The lifetime of the battery power has no impact on this system because the energy supply of the hybrid sensor is only from the electromagnetic $\mathrm{RF}$ field of the transceiver unit [8]. Actually, dual channel differential mode was adopted with two SAWR and only one of the SAWR was used as a reference, thus it could reduce the impact of temperature and pressure. This paper presents the technical scheme and implementation details of the non-destructive detection of the rebar buried in concrete walls by combining SAW resonance with self-inductive sensor. The validity of the proposed method is verified through theoretical calculation and analog simulation.

\section{PRINCIPLES}

In theory, SAW sensor can be divided into delay line mode with phase output and resonator mode with frequency output. For SAW delay line, it is an analog measurement and the measured signal is converted into time delay. Compared with measuring frequency, it is not convenient to measure the time delay which often results in low accuracy. Furthermore, the distance between remote controlling element and sensing element has a serious effect on the time delay. For SAW resonator, the inter-digital transducer is placed between two total reflecting grates. If the excited frequency of SAW is identical with inherent frequency of SAW resonator, the standing-wave of SAW is formed between reflecting grates with the maximum reflection. When one or more of the oscillation loop parameters change, the center frequency of resonator, which could be obtained as proposed in [9] and [10], will shift and the frequency deviation corresponds to the measured quantity. Fig. 1 shows the single port SAWR schematic diagram of remote sensing system. An RF wide band pulse interrogation signal is emitted and received by the antenna of the SAW transponder unit. It excites a standing-wave of SAW in resonant cavity at the central frequency of the sensor. After switching off the exciting signal, the inter- 
digital transducer connected to the antenna reflects a decaying signal to the central transceiver unit where the measured quantity is obtained from the reflected signal frequency gauged by a frequency meter.

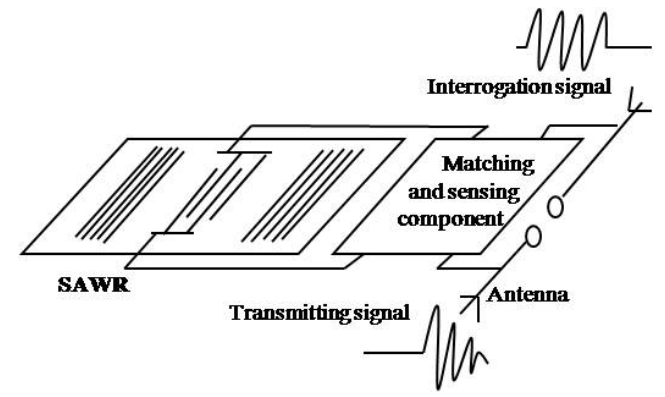

Fig.1. Passive wireless hybrid SAW non-destructive testing system

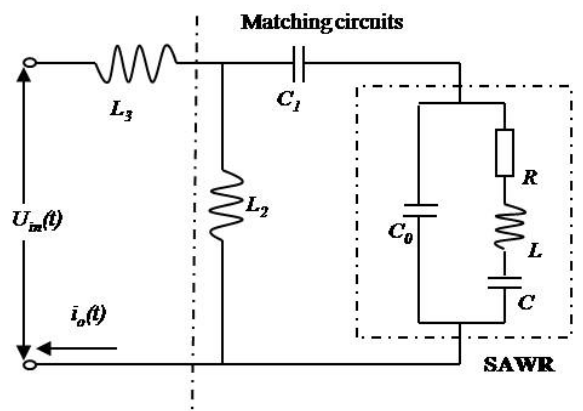

Fig.2. Equivalent circuits of SAWR and matching network

In the proposed method, the SAW device is used as the transmitter and the buried rebar is inspected by a selfinductive sensor which is connected to matching network. $\mathrm{Q}$ factor is an important physical quantity which is the ratio of stored energy of resonant circuit to dissipation energy. The higher the $\mathrm{Q}$ factor is, the smaller the loss and the higher the efficiency it has. In order to increase the $\mathrm{Q}$ factor and overcome the mismatch caused by the variation of the parameters of oscillation loop [11], the designed resonator must possess enough bandwidth and necessary measures should be taken to compensate the mismatch in the designing of matching circuit. Klett et al. proposed a solution to this problem in [12]. Firstly, the SAWR is depicted as a serial $R L C$ circuit paralleling a static SAW capacitance $C_{0}$. Secondly, in order to meet the wellmatched requirement, a reactive component is applied to make the real impedance of equivalent circuit $50 \mathrm{ohm}$. Finally, since the resonant frequency changes after using a reactance to adjust resistance, it is necessary to cascade another reactance to make the resonant frequency identical with SAWR inherent frequency. After all the above steps finished, the matching network would have high $\mathrm{Q}$ factor and low mismatch.

Fig. 2 shows the equivalent circuit of SAWR and matching network, where the capacitor $C_{1}$ is used to realize $50 \mathrm{ohm}$ condition, inductor $L_{2}$ is to adjust the resonant frequency of the system, and $L_{3}$ is sensing self-inductor which changes with respect to the depth of buried rebar. If the detection point of the reinforced concrete wall has no rebar, the value of $L_{3}$ will be close to zero. The wellmatched circuit and SAWR could be equivalent to a series $R_{e q} L_{e q} C_{e q}$ resonant circuit. After integrating the sensing inductor into the matching circuit, the reflection coefficient $S_{11}$ was:

$$
S_{11}=\frac{Z_{r}-Z_{0}}{Z_{r}+Z_{0}}=\frac{j \omega L_{3}+j \omega L_{e q}+R_{e q}+1 / j \omega C_{e q}-Z_{0}}{j \omega L_{3}+j \omega L_{e q}+R_{e q}+1 / j \omega C_{e q}+Z_{0}}
$$

The resistance $Z_{0}$ is the standard resistance with $50 \mathrm{ohm}$. The transfer function of the system can be expressed as:

$$
H(f)=\frac{I_{o}(f)}{U_{i n}(f)}=\frac{1}{j 2 \pi f L_{3}+j 2 \pi f L_{e q}+R_{e q}+1 / j 2 \pi f C_{e q}}
$$

The resonant frequency of the system $f$ and the SAWR inherent frequency $f_{0}$ are respectively as:

$$
\begin{gathered}
f=\frac{1}{2 \pi \sqrt{\left(L_{3}+L_{e q}\right) C_{e q}}} \\
f_{0}=\frac{1}{2 \pi \sqrt{L C}}=\frac{1}{2 \pi \sqrt{L_{e q} C_{e q}}}
\end{gathered}
$$

According to (2), the changes of $L_{3}$ would inevitably result in changes of the amplitude and phase of the transfer function $H(f)$. Thus, there are three signal demodulation methods which are based on adjusting resonant frequency, amplitude and phase, respectively. The demodulation of resonant frequency is the most accepted method due to its simplicity, good stability and restraining of noise. Two SAW resonators with the same parameter and working under the same environment are adopted to improve the precision and reduce the difficulty in signal processing. One of the SAWR is used to detect rebar and the other one is used as the reference. The difference in the frequencies between these two resonators is caused by the rebar buried within the reinforced concrete walls. The difference of these two SAWR outputs decreases the frequency of the detected signal, and reduces the influence of common mode noise such as environment temperature, humidity, etc., thus it can improve the reliability of the results.

\section{Simulation OF THE RELATION BETWEEN THE DEPTH OF REBAR AND THE TRANSFER FUNCTION}

For a self-inductive sensor as shown in Fig.3, the core magnetic reluctance $R_{F}$ is very small but the leakage reluctance $R_{\text {leak }}$ is very large, thus the reluctance of the magnetic circuit is: 


$$
R_{m}=\left(R_{F}+R_{\delta}\right) / / R_{\text {leak }} \approx R_{\delta}=\frac{2 \delta}{\mu_{0} A}
$$

$A$ is the air gap cross sectional area of the magnetic circuit, $\delta$ the length of air gap, and $\mu_{0}=4 \pi \times 10^{-7}$ is the magnetic permeability of vacuum.

The working principle of the self-inductive sensor for detection of the buried rebar in concrete walls is that the depth of embedded rebar is equivalent to the air gap length. If the sensor moves along the wall where the buried rebar exists, the variation of the depth of embedded rebar would result in the proportional variation of magnetic reluctance.

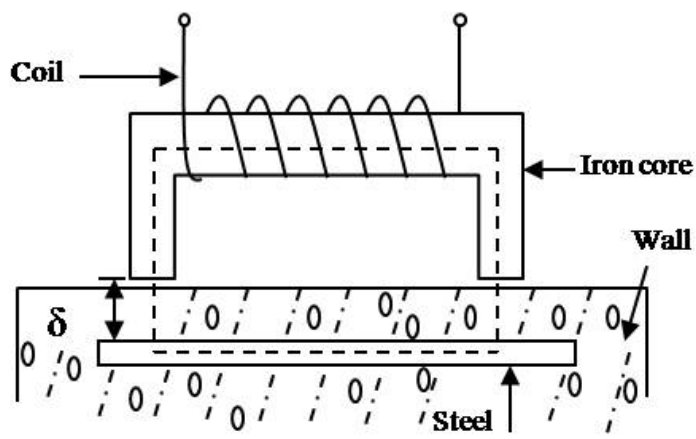

Fig.3. The structure of self-inductive sensor

The magnetic reluctance and inductance have an inverse relation; hence the value of $L_{3}$ changes as follows:

$$
L_{3}=\frac{N^{2}}{R_{m}}=\frac{N^{2} \mu_{0} A}{2 \delta}
$$

Where $N$ is the turning number of the coil. For a given sensor, $N$ and $\mu_{0}$ are constant. According to values of $L_{3}$, we know whether there is a buried rebar or not in the inspected area, and also know the depth of the buried rebar. If $N=10, A=2 \mathrm{~cm}^{2}, \delta=5 \mathrm{~cm}$, then $L_{3}(251.33 \mathrm{nH})$ amount to $n H$ order of magnitude which was far less than $L_{e q}$ compared with the equivalent inductance of SAWR. Therefore, the frequency deviation is:

$$
\Delta f=f-f_{0} \approx f_{0}\left(1-\frac{L_{3}}{2 L_{e q}}\right)-f_{0}=-\frac{N^{2} \mu_{0} A}{4 \delta L_{e q}} f_{0}
$$

According to (7), the frequency deviation is proportional to the sensing inductance and the shift of frequency within the bandwidth limit. Thus, the system works close to SAWR inherent frequency with high $\mathrm{Q}$ factor and low mismatch. After rationally designing the inductance and capacitance of matching network, a kind of non-destructive detecting approach was developed to inspect the buried rebar in concrete walls by combining SAWR device with selfinductive sensor.

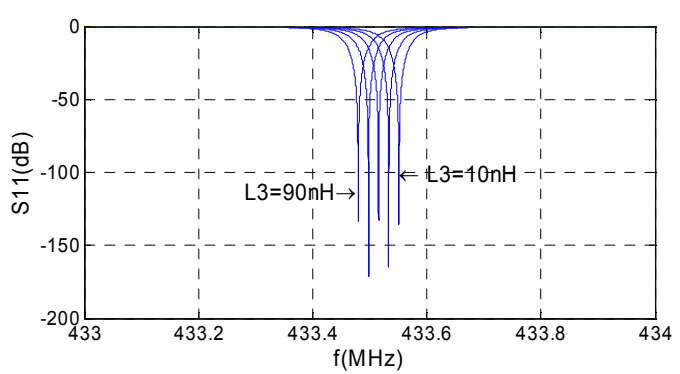

(a) The reflection coefficients with different $L_{3}$

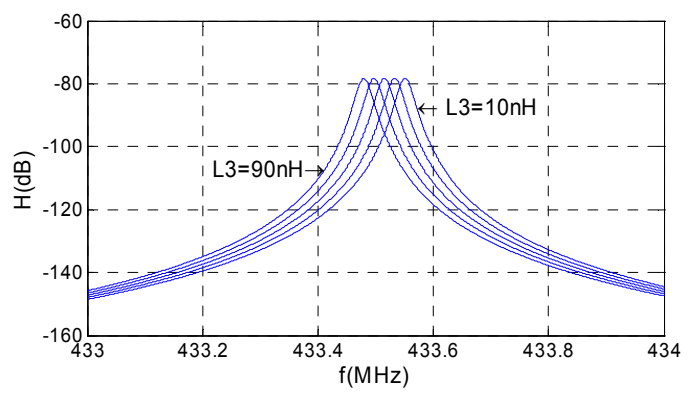

(b) The transfer functions with different $L_{3}$

Fig.4. Effects on the reflection coefficient $S_{11}$ and transfer function $H(f)$ with varying inductor $L_{3}$

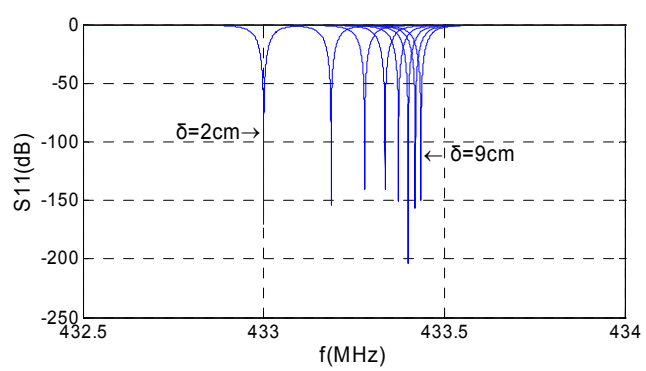

(a) The reflection coefficient with different $\delta$

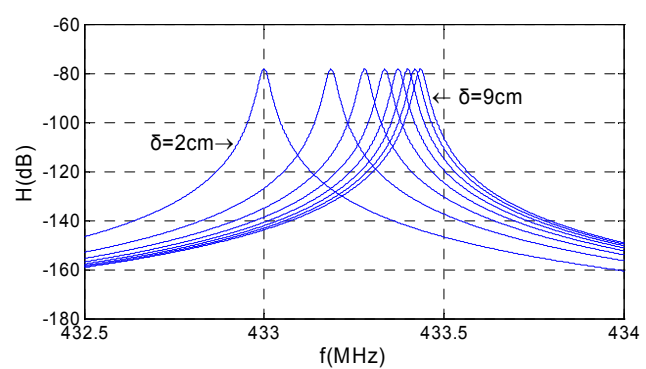

(b) The transfer function with different $\delta$

Fig.5. Effects on the reflection coefficient $S_{11}$ and transfer function by varying the depth $\delta$

The parameters are set as follows: $R_{0}=1.55 \Omega$, $C_{0}=3.76 \mathrm{pF} \quad, \quad R=12.85 \Omega \quad, \quad L=65.83 \mu \mathrm{H} \quad$ ， $C=2.05 \mathrm{fF}, f_{\mathrm{s}}=433.489 \mathrm{MHz}$, so the equivalent $R L C$ are: $R_{e q}=50.1 \Omega, L_{e q}=242.8 \mu \mathrm{H}, C_{e q}=0.555 \mathrm{fF}$. If $L_{3}$ varies between $10 \mathrm{nH}$ and $90 \mathrm{nH}$, the effects on reflection coefficient $S_{11}$ and transfer function $H(f)$ are 
shown in Fig.4. As can be seen from Fig.4, the larger the inductance $L_{3}$ is, the smaller the resonant frequency is and it remains close to the SAWR inherent frequency and meets the requirements of high $\mathrm{Q}$ and low mismatch. If there is no rebar buried in the detecting area, the system resonant frequency is approximately equal to SAWR inherent frequency in terms of (3) and (4) because $L_{3}$ is close to zero. For another circumstance, in order to confirm the depth of rebar, it is necessary to move the sensor within a small range to find the minimum frequency. Since the depth of buried rebar changes minimally in a small range, $L_{3}$ is affected mostly by the effective cross sectional area and the maximum $L_{3}$ corresponds to the minimum resonant frequency. Considering that the effective cross sectional area between buried rebar and iron core keep with the maximum constant, the depth of buried rebar could be obtained with (7).

When $\delta$ changes from $2 \mathrm{~cm}$ to $9 \mathrm{~cm}$, its effects on the reflection coefficient $S_{11}$ and transfer function $H(f)$ are shown in Fig. 5 with the effective cross sectional area at maximum constant $2 \mathrm{~cm}^{2}, N=10$. It can be seen that a small depth corresponds to a large frequency deviation and the resonant frequency moves closer to the SAWR inherent frequency when the depth becomes larger.

\section{CONCLUSION}

A new wireless passive non-destructive method for detection of the rebar embedded within reinforced concrete walls is presented by combining a self-inductive sensor with a SAW device. The system has the advantages of high $Q$ value and low mismatch after rationally designing the matching circuit. The validity of inspecting the buried rebar is verified through theoretical calculation and simulation. With the depth of the buried rebar becoming larger, the frequency deviation, from which the depth could be deduced using the signal processing system, becomes smaller. However, if the buried rebar is close to the reinforced concrete wall surface, the resonant frequency will shift from SAWR inherent frequency and result in mismatch. So it is important to design a converter circuit to make the inductance amount to $\mathrm{nH}$ order of magnitude. The system would result in the misjudgment when the wall is made from large magnetic permeability material, and/or the buried rebar has different diameters. Further work will focus on solving the existing problems and doing the experiments based on the theoretical analysis.

\section{ACKNOWLEDGMENT}

This work was supported by the Natural Science Foundation of China, under Grant No.50875167

\section{REFERENCES}

[1] Valíček, J., Držík, M., Hryniewicz, T., et al. (2012). Non-Contact method for surface roughness measurement after machining. Measurement Science Review,12 (5), 184-188.
[2] Hola, J., Schabowicz, K. (2005). Application of artificial neural networks to determine concrete compressive strength based on non-destructive tests. Journal of Civil Engineering and Management, 11 (1), 23-32.

[3] Diego, S.B., Sung, Q., Patrich, G.A., et al. (2008). A preliminary magneto inductive sensor system for realtime imaging of steel reinforcing bars embedded within concrete. IEEE Transactions on Instrumentation and Measurement, 57 (11), 2437-2442.

[4] Popovics, J.S., Gallo, G.E., Shelton, M., et al. (2007). A magnetic sensing approach to characterize corrosion in reinforced concrete. In Sensors and Smart Structures Technologies for Civil, Mechanical, and Aerospace Systems 2007, Proceedigs of SPIE, vol. 6529.

[5] Tsukada, K., Kiwa, T., Kawata, T., et al. (2006). Lowfrequency eddy current imaging using MR sensor detecting tangential magnetic field components for nondestructive evaluation. IEEE Transactions on Magnetics, 42 (10), 3315-3317.

[6] Yamazaki, K., Ishikawa, K., Haga, A., et al. (2010). Impedance measurement using a resonance circuit for detecting steel bars and cables inside pliable plastic conduit tubes buried in concrete walls and slabs. IEEE Transaction on Magnetics, 46 (6), 1963-1966.

[7] Binhack, M., Buff, W., Klett, S., et al. (2000). A combination of SAW-resonators and conventional sensing elements for wireless passive remote sensing. In 2000 IEEE Ultrasonics Symposium, 22-25 October 2000. IEEE, 495-498.

[8] Schimetta, G., Dollinger, F., Weigel, R., et al. (2000). A wireless pressure measurement system using a SAW hybrid sensor. In 2000 IEEE MTT-S International Microwave Symposium Digest, 11-16 June 2000. IEEE, vol. 3, 1407-1410.

[9] Petrović, P. (2012). Frequency and parameter estimation of multi-sinusoidal signal. Measurement Science Review, 12 (5), 175-183.

[10] Angrisani, L., Capriglione, D., Ferrigno, L., Miele, G. (2011). A new digital signal processing method for spectrum interference monitoring. Measurement Science Review, 11 (1), 1-8.

[11] Fu, Q., Wang, J., Luo, W., et al. (2008). Progress of matching network for passive remote hybrid sensor based on SAW resonator. In IUS 2008 : IEEE Ultrasonics Symposium, 2-5 November 2008. IEEE, 1512-1515.

[12] Klett, S., Blinhack, M., Buff, W., et al. (2002). Progress in modeling of sensor function for matched SAW resonators. In 2002 IEEE Ultrasonics Symposium, 8-11 October 2002. IEEE, 471-474.

Received June1, 2012. Accepted January 28, 2013. 